\title{
Structurally constrained protein evolution: results from a lattice simulation.
}

\author{
Ugo Bastolla ${ }^{(1)}$, Michele Vendruscolo ${ }^{(2)}$ and H. Eduardo Roman ${ }^{(3)}$ \\ ${ }^{(1)}$ FU Berlin, Inst. für Kristallographie, Takustr. 6, D-14195 Berlin, Germany \\ ${ }^{(2)}$ Department of Physics of Complex Systems, Weizmann Institute of Science, Rehovot 76100, Israel \\ ${ }^{(3)}$ INFN, Sezione di Milano, Università di Milano, Via Celoria 16, I-20133 Milano, Italy
}

\begin{abstract}
We simulate the evolution of a protein-like sequence subject to point mutations, imposing conservation of the ground state, thermodynamic stability and fast folding. Our model is aimed at describing neutral evolution of natural proteins. We use a cubic lattice model of the protein structure and test the neutrality conditions by extensive Monte Carlo simulations. We observe that sequence space is traversed by neutral networks, i.e. sets of sequences with the same fold connected by point mutations. Typical pairs of sequences on a neutral network are nearly as different as randomly chosen sequences. The fraction of neutral neighbors has strong sequence to sequence variations, which influence the rate of neutral evolution. In this paper we study the thermodynamic stability of different protein sequences. We relate the high variability of the fraction of neutral mutations to the complex energy landscape within a neutral network, arguing that valleys in this landscape are associated to high values of the neutral mutation rate. We find that when a point mutation produces a sequence with a new ground state, this is likely to have a low stability. Thus we tentatively conjecture that neutral networks of different structures are typically well separated in sequence space. This results indicates that changing significantly a protein structure through a biologically acceptable chain of point mutations is a rare, although possible, event.
\end{abstract}

\section{INTRODUCTION}

Almost unmistakingly, naturally occurring proteins with sequence similarity larger than $40 \%$ adopt similar folds [1]. Since natural proteins with homologous sequences descend from a common ancestor, this observation indicates that protein structures are significantly conserved in evolution. Indeed, several proteins with different functions show a remarkable structural similarity of evolutionary origin even if their sequences can not anymore be recognized as related [2,3]. A recent study on the Protein Data Bank (PDB) showed that the typical sequence similarity between proteins with the same fold is about $8.5 \%$ [A], only slightly larger than for a random pair of sequences [5]. In this set also proteins with common ancestors are likely to exist. These observations cue to the fact that during evolution, there is a strong memory for the structure but only a very loose memory for the sequence.

The neutral theory of molecular evolution, proposed in 1968 by Kimura [6] and, independently, by Jukes and King [7], is well consistent with these observations. Kimura suggested that most amino acid substitutions in protein sequences are selectively neutral, i.e. indistinguishable from the wild type from the phenotypic point of view, and are fixed by chance in biological populations [6]. This hypothesis has been heatedly debated in the genetic literature 88.97. Strictly speaking, conservation of the fold and neutral evolution are not equivalent, since neutrality deals with the activity of the protein, concentrated in its active site, more than with its structure. Moreover drastic changes in the environment can mod- ify the selective value of protein structures. However, since our model does not represent biological activity, we assume in the following neutrality to be synonymous of structure conservation.

In the last decade, the possible occurrence of neutral evolution has been revealed by a series of computational and analytic studies of the sequence to secondary structure relationship for RNA molecules [10]. An exponentially large number of sequences correspond on average to a single structure, and the distribution of the number of sequences per structure is quite broad (following a power law), with the most common structures forming connected neutral networks which percolate sequence space.

For proteins, the sequence to structure relationship is much more difficult to study than for RNA. Shakhnovich and Gutin [11], using the Random Heteropolymer model, argued that the probability that a point mutation is neutral (i.e. it does not alter the native state) is non vanishing even for very long sequences. In the same spirit, Tiana et al. 12] considered a cubic lattice model and a sequence with 36 residues, optimized in such a way that its ground state coincides with a target structure and is very stable [13, and estimated that $70 \%$ of the point mutations are neutral. Bornberg-Bauer 14 studied a two-dimensional HP model with only two residue types and chain length $N=18$ by using exact enumeration. He found, in analogy with the RNA case, that the distribution of the number of sequences per structure is very broad, but sequences corresponding to the same structure are clustered in small regions of sequence space.

We simulate the evolution of a protein sequence sub- 
ject to structure conservation. Mutations that change the protein's native structure, identified with the ground state of the model, are considered lethal and are rejected. In this way, our sequence follows an evolutionary trajectory on a neutral network, i.e. a set of sequences sharing the same fold and connected by point mutations. While the structure (fold) is conserved, the sequence changes as new mutations are accepted, and after a sufficient number of steps along the evolutionary trajectory have been performed, the sequence behaves essentially as a random one with respect to the original one [15].

It is important, however, to impose not only the condition that the native state is conserved but also that its stability remains high and the folding time remains low. These conditions are not only biologically relevant, but also help the model protein to diffuse in sequence space.

We use in this study a lattice representation of protein conformations, because only in this way the ground state and its thermodynamic stability can be reliably determined, but we believe that our simplified model reproduces the generic features of the evolution of real protein sequences.

Support to our results comes from a recent study by Babajide and coworkers [16], who found evidence for the presence of neutral networks in protein sequence space. Their work is similar in spirit to the present one, but rather different methodologically. Real protein structures were represented through the $C_{\alpha}$ and $C_{\beta}$ coordinates taken from the PDB, and an approximate criterion of fold recognition based on the $\mathrm{Z}$ score [17] was used. Further support also comes from the work of Govindarajan and Goldstein [18,19], who introduced the "foldability" landscape in order to describe molecular evolution. In the language of Govindarajan and Goldstein the foldability of a protein represents its fitness for survival during evolution and it is related to the stability and to the kinetic accessibility of the native state. Govindarajan and Goldstein also found that their evolutionary dynamics in sequence space was confined inside "neutral networks".

Tha main result of our paper, namely the fact that the fraction of neutral neighbors strongly fluctuates inside the neutral network, and that these fluctuations can be related to the foldability landscape, should also be put in relation with the recent preprint by Tiana et al. 20. where it is shown that the energy of a target structure has a complex landscape with valleys and barriers in sequence space. The present work supports such a picture by using the same protein model but employing rather different methods of investigation.

We already presented some results on neutral networks in Ref. [15]. Here we focus our attention on the issue of the stability of the native state, relating it to the characteristics of the evolution. In Sec.II, we describe our model protein and our protocol to simulate neutral evolution. In Sec.III we summarize our previous results. In Sec.IV we describe the properties of the sequences generated, dividing them in four classes. This section focuses on the relation between thermodynamic stability and evolu- tionary dynamics. Sec. $\mathrm{Q}$ presents an overall discussion, relating our results to biological observations.

\section{A SIMPLE MODEL OF PROTEIN EVOLUTION}

In this section we define the lattice model used to represent protein structure and the algorithm introduced to simulate evolution in sequence space.

\section{A. Lattice model of protein structure}

To investigate the correspondence between sequences and structures we use a lattice model with twenty amino acid types. We consider sequences of length $N=36$, denoted by the symbol $\mathbf{S}=\left\{s_{1}, \ldots, s_{N}\right\}$, where $s_{i}$ belongs to a twenty-letter alphabet. Configurations are represented by self avoiding walks on the simple cubic lattice, where each occupied site represents an amino acid. An energy $E(\mathbf{S}, \mathcal{C})$ is assigned to configuration $\mathcal{C}$ of sequence $\mathbf{S}$ according to the rule:

$$
E(\mathbf{S}, \mathbf{C})=\sum_{i<j}^{1, N} C_{i j} U\left(s_{i}, s_{j}\right)
$$

where $U(a, b)$ is a $20 \times 20$ symmetric interaction matrix expressing the contact interactions of amino acids of species $a$ and $b$. We use an interaction matrix $U(a, b)$ derived from the Miyazawa-Jernigan interaction matrix 21]. The matrix $\mathbf{C}=\left\{C_{i j}\right\}=f(\mathcal{C})$, called the contact map of configuration $\mathcal{C}$, has elements $C_{i j}$ equal to one if residues $i$ and $j$ are nearest neighbors on the lattice but not along the chain and zero otherwise. The similarity between contact maps is measured through the overlap $q\left(\mathbf{C}, \mathbf{C}^{\prime}\right)$, defined as

$$
q\left(\mathbf{C}, \mathbf{C}^{\prime}\right)=\frac{1}{N_{c}^{*}} \sum_{i<j} C_{i j} C_{i j}^{\prime},
$$

where $N_{c}^{*}$ is the maximal between $N_{c}$ and $N_{c}^{\prime}$, the number of contacts respectively of two contact maps $\mathbf{C}$ and $\mathbf{C}^{\prime}$, and $N_{c}=\sum_{j>i} C_{i j}$. With this definition, two maps are identical if and only if $q=1$. Note that this does not imply in general identity of configurations. Nevertheless, we use the overlap as a measure of similarity in configuration space because structures with the same contact map are degenerate in energy and for compact structures, only small conformational fluctuations are allowed when the entire set of contacts is specified. Moreover, such structural fluctuations might play an important role in protein functionality [22].

The native structure of sequence $\mathbf{S}$ is identified with the ground state of the model if it is thermodynamically stable. We evaluate stability by measuring the average overlap $\langle q\rangle$ between the ground state and the Boltzmann ensemble of structures: 


$$
\langle q\rangle=\frac{1}{Z} \sum_{\mathcal{C}} q\left(\mathbf{C}_{0}, f(\mathcal{C})\right) e^{-E(\mathcal{C}, \mathbf{S}) / T},
$$

where $\mathbf{C}_{0}$ is the contact map of the ground state, $f(\mathcal{C})$ is the contact map of configuration $\mathcal{C}$ and $Z$ is the partition function. This quantity is close to one if all the low energy structures are quite similar to the native state. In this case the energy landscape of the model is well correlated, and the sequence is also expected to be a good folder.

FIG. 1. The "native state" of the model protein. The initial residue is the one in the bottom left corner.

We consider the target contact map $\mathbf{C}^{*}$ represented in Fig.1. It has $N_{c}=40$ contacts, the maximal number of contacts possible for a chain of length $N=36$. In this case the contact map defines uniquely the configuration of the system. The contact map $\mathbf{C}^{*}$ was studied by Shakhnovich and coworkers in a computer experiment of inverse folding 13]. They designed a sequence with ground state on $\mathbf{C}^{*}$ using the procedure of Ref. [23], and showed that $\mathbf{S}^{*}$ has good properties of kinetic foldability and thermodynamic stability at the temperature where the folding is fastest. The lower part of the energy landscape of this sequence is remarkably smooth: all the structures with low energy have a high overlap $q$ with the ground state. The lowest energy of configurations with a fixed value of $q$ decreases regularly as $q$ approaches one. This correlated energy landscape, reminiscent of the "funnel" paradigm [24], is the reason of the good folding properties of the sequence, which is very different from a random sequence. In 12] it was shown that the same sequence is also very stable against mutations. It was estimated that about $70 \%$ of the point mutations performed on $\mathbf{S}^{*}$ result in new sequences with exactly the same ground state and good folding properties. Thus energy minimization makes $\mathbf{C}^{*}$ stable not only in structure space, but also in sequence space.

We note that $\mathbf{C}^{*}$ is an atypical structure for the interaction parameters that we choose: since $U(a, b)$ has average value zero and variance 0.3 , one would expect open structures to be energetically favored. Indeed, typical random sequences with $N=36$ and Gaussian contact interactions whose average vanishes have a ground state with approximately 29-33 contacts [25], being thus less than maximally compact.

\section{B. Sequence space}

In this study we consider only point mutations, thus all sequences have the same length $N=36$ and the metric in sequence space is given by the Hamming distance,

$$
D\left(\mathbf{S}, \mathbf{S}^{\prime}\right)=\sum_{i=1}^{N}\left[1-\delta\left(s_{i}, s_{i}^{\prime}\right)\right],
$$

where $\delta$ is the Kronecker symbol and $s_{i}$ takes 20 different values, one for each amino acid. A measure of sequence similarity is then given by the overlap $Q\left(\mathbf{S}, \mathbf{S}^{\prime}\right)$,

$$
Q\left(\mathbf{S}, \mathbf{S}^{\prime}\right)=\frac{1}{N} \sum_{i=1}^{N} \delta\left(s_{i}, s_{i}^{\prime}\right),
$$

which is equal to one minus the normalized Hamming distance.

We introduce also the distance $D_{H P}\left(\mathbf{S}, \mathbf{S}^{\prime}\right)$ and the overlap $Q_{H P}\left(\mathbf{S}, \mathbf{S}^{\prime}\right)$ to measure differences in hydrophobicity. These are defined by transforming every sequence into a sequence of binary symbols, either $\mathrm{H}$ or $\mathrm{P}$, according to the hydrophobicity of the residue. We consider 8 hydrophobic amino-acids and 12 polar ones. The definitions of $D_{H P}$ and $Q_{H P}$ are analogous to those of $D$ and $Q$, where now $s_{i}$ can take only two values.

\section{Evolutionary process}

Our protein sequence evolves through point mutations subject to conservation of the target contact map $\mathbf{C}^{*}$, representing the biologically active native structure [26]. We impose this condition by simulating the following iterative procedure:

1. At $t=0$ we start from $\mathbf{S}(0)=\mathbf{S}^{*}$, which has $\mathbf{C}^{*}$ as its "native state".

2. At time $t$ we mutate at random one amino acid in $\mathbf{S}(t-1)$, producing a new sequence $\mathbf{S}^{\prime}(t)$.

3 . We submit the new sequence to selection according to the criteria specified below. If the sequence is accepted then $\mathbf{S}(t)=\mathbf{S}^{\prime}(t)$, otherwise we restore $\mathbf{S}(t)=\mathbf{S}(t-1)$.

The selection step is governed by the following three conditions

Conservation of the ground state. The ground state $\mathbf{C}$ of $\mathbf{S}^{\prime}(t)$ must have an overlap with $\mathbf{C}^{*}$ equal or larger than a given "phenotypic threshold" $q_{\mathrm{thr}}$.

$$
q\left(\mathbf{C}^{*}, \mathbf{C}\right)>q_{\mathrm{thr}},
$$

In our calculations we imposed strict conservation of the native state by setting $q_{\mathrm{thr}}=1$.

Thermodynamic stability . We define thermodynamic stability through the condition

$$
\left\langle q\left(\mathbf{C}^{*}, \mathbf{C}\right)\right\rangle>\langle q\rangle_{\mathrm{thr}}
$$

where $\langle\cdot\rangle$ represent a Boltzmann average at the temperature $T$ of the simulation and $\langle q\rangle_{\mathrm{thr}}$ is a fixed parameter. This condition implies that all the thermodynamically relevant states are very similar to the target state. 
Kinetic accessibility . The structure $\mathbf{C}^{*}$ must be reached in a limited number of steps of our Monte Carlo algorithm, in at least two independent attempts.

For the test of the sequences we used the PERM method [27,28, a Monte Carlo algorithm particularly suited for finding the ground state of lattice polymers. Note that there is no bias towards $\mathbf{C}^{*}$ in our Monte Carlo algorithm, i.e. it has the same a priori probability of being visited as any other possible structure. We remark that other schemes of simulations are also suitable to the same effect, as e.g. the Monte Carlo algorithm used in Ref. [29]. Such Monte Carlo method with moves in configuration space is more suitable than PERM to estimate folding times. However, due to computational limitations, we did not try to measure accurately the folding time, thus we adopted the PERM method, which is faster for the task that is interesting for us.

The test of a new sequence $\mathbf{S}$ is divided into three phases:

- We discard $\mathbf{S}$ if after $m$ iterations $\mathbf{C}^{*}$ is not reached or if other structures of energy lower than $\mathbf{C}^{*}$ are found.

- Otherwise we continue to run the algorithm for another $m$ iterations and discard $\mathbf{S}$ if we find structures of energy lower than $\mathbf{C}^{*}$.

- If $\mathbf{S}$ passed the first two phases, we run again and independently the MC algorithm for a time $2 m$ and accept $\mathbf{S}$ if also this time $\mathbf{C}^{*}$ is found as the lowest energy structure.

Thus for each accepted sequence we run the algorithm for $4 m$ steps, with $m=124000$. We never found in the second independent run of the MC algorithm a structure with lower energy than the putative ground-state $\mathbf{C}^{*}$ found in the first run. This fact encourages us to believe that the algorithm was effective in finding the ground state. Another support to this conclusion comes from the fact that, as it will be discussed later in more detail, all of the selected sequences have a remarkably correlated energy landscape, which makes the task of finding the ground state easier.

On the other hand, whenever the sequence was rejected, we are less sure that we were able to determine its ground state. The difference is due to two reasons: first, we investigate rejected sequences on the average for a shorter time. Second, rejected sequences have typically a less correlated energy landscape, so that the determination of the ground state should be more difficult. Nevertheless, we shall present in SecIV also data about rejected sequences, since they are interesting and refer to a very large number of sequences, even if they are individually not completely reliable.

The three conditions for the acceptance of a mutation enforce the conservation of the fold of the protein. This is similar to neutral evolution where the biological activity of the mutated sequence does not vary. Nevertheless, conservation of the fold is not a necessary condition for selective neutrality in real proteins, although a very high degree of conservation is usually observed, and it is not even a sufficient one, since - in the case of enzymes the active site has also to be conserved and the environment has to remain reasonably stable. Thus our model represents the neutral evolution of the part of the chain not involved in chemical activity, in a stable chemical environment. Despite its simplifications, we believe that our model captures important features of structural constraints in the neutral evolution of proteins.

\section{NEUTRAL NETWORKS}

In this section we summarize results regarding the diffusion in sequence space under our model of neutral evolution. More details have been given in Ref. [15].

\section{A. Hamming distance}

An interesting result of our simulation is that sequences originated from the same common ancestor diverge so much that their similarity is almost as low as for a random pair of sequences while their structures remain unchanged. Starting from the same sequence we generated eight realizations of neutral evolution, simulating the phylogenetic radiation of eight species from a common ancestor. We use the following values of the selection parameters: phenotypic threshold $q_{\mathrm{thr}}=1$, corresponding to exact conservation of the ground state, stability threshold $\langle q\rangle_{\mathrm{thr}}=0.90$ at a temperature $T=0.16$ chosen so that the folding of the initial sequence $\mathbf{S}^{*}$ is fastest with our Monte Carlo algorithm.

The average Hamming distance between the final points of the eight evolutionary trajectories is $D=30.2$, only slightly smaller than the random value $D^{r a n}=34.2$ (see Fig.2). However, this quantity had not yet reached a stationary value when the simulations were interrupted, thus we can not exclude that the long time behavior coincides with $D^{r a n}$. An indication in this sense is the fact that the maximum distance between sequences in two different trajectories is $D=35$. All the residues in the original sequence could be substituted at least twice, but some are more difficult to change. We define the degree of conservation, or rigidity, of residues at the $i$-th position in the sequence as follows:

$$
R_{i}=\sum_{a} P_{i}^{2}(a)
$$

where $P_{i}(a)$ is the probability to find the amino-acid $a$ at position $i . P_{i}(a)$ is estimated from the end points of the eight neutral paths generated. $R_{i}=1$ if the aminoacid at position $i$ is never changed, while $R_{i} \approx 1 / 8$ if 
it is completely random. We found that several positions have rigidity compatible with the random value, and no position has $R_{i}=1$. As one would expect, the most conserved positions are the two in the interior of the structure (see Fig.2).

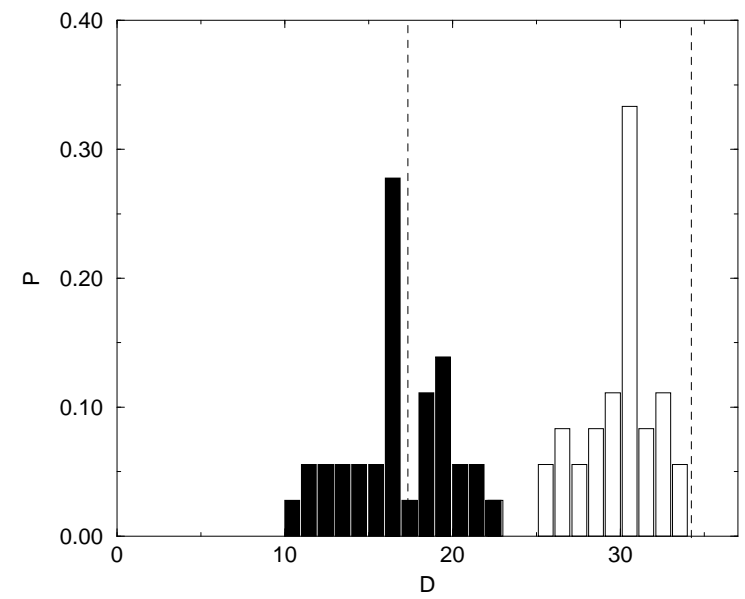

FIG. 2. Histograms of the Hamming distances between the end points of eight independent trajectories using the full 20 amino-acid alphabet (white) and the reduced HP alphabet (black). The vertical dashed lines represent the average values for random pairs of sequences.

The same results hold for the HP representation in which hydrophobic $(\mathrm{H})$ and polar $(\mathrm{P})$ amino acids are grouped together so that $s_{i}$ can assume only two values. The average value of the distance is in this case $D_{H P}=16.3$, not far from the random value $D_{H P}^{r a n}=17.3$, and the variance is $V_{H P}=6.0$, compatible with $V_{H P}=$ $D_{H P}\left(1-D_{H P}\right) / N$. It is at first sight surprising that also the distance $D_{H P}$ is close to that expected for random sequences. This is in part an effect of the short length of our sequences, since only two residues are in the interior of the structure, while all other ones are at the surface. It is interesting to note, however, that also the two residues in the interior of $\mathbf{C}^{*}$ have rigidity $R_{i}<1$, even when the two letter HP representation is used. The distinction between polar and hydrophobic residues is based only on the interaction matrix that we use, in which also polar residues can have attractive interactions. It is interesting to note that a recent study of real protein structures 30 found a correlation between amino acids buried in the core and evolutionary conserved ones, consistently with our results.

\section{B. Neutral Mutation Rate}

For a given sequence $\mathbf{S}$ of $N$ amino acids, we define the neutral mutation rate $x(\mathbf{S})$ as the fraction of acceptable non-synonymous mutations

$$
x(\mathbf{S})=\frac{1}{20 N} \sum_{i=1}^{N} \sum_{\alpha \neq s_{i}}^{1,20} \chi_{\alpha i}(\mathbf{S}),
$$

where $\chi_{\alpha i}(\mathbf{S})$ equals one if assigning the amino acid of species $\alpha$ at position $i$ on the sequence $\mathbf{S}$ does not change the native state, and zero otherwise. Non-synonymous mutations are those for which an amino acid is not replaced by itself.

The simplest measure of the neutral mutation rate is obtained by computing the frequency of neutral mutations over all the non-synonymous mutations proposed. In this way we found $\bar{x} \approx 0.05$ (the overline represents an average over the mutational process). However, this quantity alone is not enough to characterize $x(\mathbf{S})$, which fluctuates strongly in sequence space. For instance, it was estimated by one of us and coworkers 12 that $x\left(\mathbf{S}^{*}\right) \approx 0.7$, where $\mathbf{S}^{*}$ is the starting point of our evolutionary trajectories.

We measured indirectly the distribution of $x(\mathbf{S})$ in sequence space from the distribution of the "trapping" time $\tau_{t}(\mathbf{S})$ that a trajectory spends on sequence $\mathbf{S}$. The average value of the trapping time is inversely proportional to the neutral mutation rate:

$$
\overline{\tau_{t}(\mathbf{S})}=\frac{1}{x(\mathbf{S})},
$$

where the bar denotes average over the different mutations. The distribution of $\tau$ at fixed $x$ is a geometric one, $P_{x}(\tau)=x(1-x)^{\tau-1}$, so that, averaging over the neutral set, we get

$$
[P(\tau)]=\int_{0}^{1} d x p(x)\left(\frac{x}{1-x}\right)(1-x)^{\tau},
$$

where [.] denotes an average over sequences belonging to the neutral network (in this argument we neglect the error in evaluating whether a sequence belongs to the neutral set: in particular, the conditions of fast folding and of thermodynamic stability are subject to considerable evaluation errors).

The distribution of $\tau_{t}$ is broader than an exponential one (Fig.3), thus, even if we can not invert Eq. 11, we expect that the distribution of the neutral mutation rate $x$ is also broader than exponential.

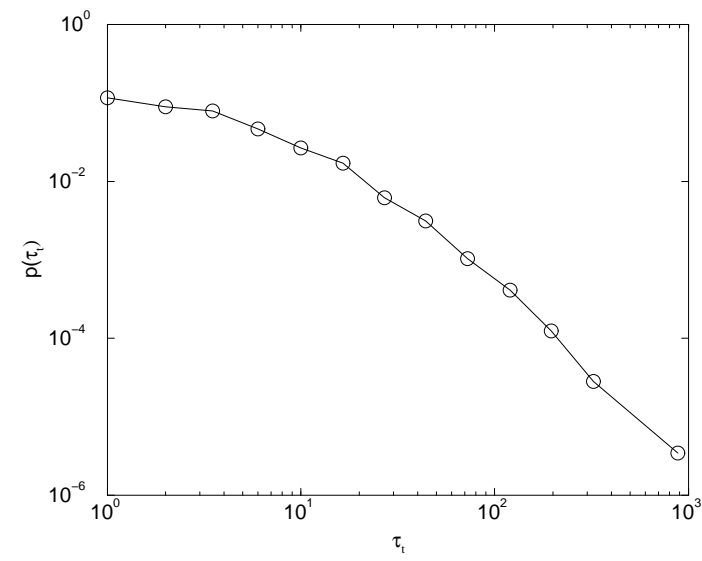


FIG. 3. Distribution of the trapping time $\tau_{t}$.

The values of $\tau_{t}$ for neighboring sequences are rather correlated, but the correlation seems to vanish after few steps in sequence space (data not shown).

\section{Genetic drift and population genetics}

In Kimura's neutral theory [6] it is assumed that the fraction of neutral mutations $x(\mathbf{S})$ does not depend on the sequence. With this hypothesis, the time evolution of the Hamming distance $D(t)$ between the starting sequence $\mathbf{S}(0)$ and the present sequence $\mathbf{S}(t)$ is given in our model by

$$
D(t) / N \approx\left(1-\frac{1}{20}\right)[1-\exp (-x t / N)]
$$

where the time $t$ represents the number of mutational events. However, this hypothesis is contradicted by our results, which show that the relaxation of the distance is not exponential. This fact is due to the large fluctuations of the neutral mutation rate along the neutral network.

This qualitative result can be interesting for the understanding of protein sequence evolution. A key issue in the theory of molecular evolution is the following: Is the rate at which amino acids are substituted in protein sequence constant on different branches of the phylogenetic tree? The constancy of the substitution rates was proposed by Zuckerkandl and Pauling in their pioneering study of molecular evolution as the molecular clock hypothesis [31. This hypothesis has been questioned recently [8.9], even if it seems to be at least approximately valid for several proteins.

Kimura's theory predicts that neutral substitutions occur at a rate $r=\mu x$ which is the product of the bare mutation rate $\mu$ times the fraction $x$ of neutral mutations. This rate is independent of the size of the population. The value $x$ characterizes the substitution rate of a particular protein but does not vary during evolution. Mutations occuring in a geological time $T$ are thought to follow a Poissonian statistics with average value $\mu T$, so that the number of substitutions is predicted to have Poissonian statistic with average value $\left\langle n_{S}\right\rangle=\mu T x$. This has the important consequence that the fluctuations of the substitution process in different species should increase only as $\sqrt{T}$. More precisely, the ratio $R(T)$ between the variance and the average value of the number of substitutions should be identically equal to one. This strong prediction was first tested by Kimura [6] with the conclusion that deviations from the Poissonian statistics are small. However, more recently Gillespie repeated the test for a larger number of proteins, finding that for most of them the value of $R(T)$ is much larger than one. He thus argued that the hypothesis that most mutations are neutral has to be rejected.
Our results provide an alternative explanation: the strong fluctuations of the substitution process can be attributed to the fluctuations of the neutral mutation rate in sequence space, even in the absence of any selective pressure. To test this hypothesis, we assume, as above, that the number $m_{k}(T)$ of attempted mutation events in a time $T$ during trajectory $k$ is a Poissonian variable of average value $\mu T$. In the present study, $k=1, \ldots 8$ is the label of the evolutionary trajectories. Then for every trajectory $k$ we count the number $n_{k}(T)$ of mutations accepted over $m_{k}(T)$ steps of our evolutionary algorithm. This number is then interpreted as the number of substitutions in "species" $k$. We can thus compute the variance and the average value of this variable over the eight trajectories. The ratio between them gives an estimate of the dispersion ratio $R(T)$. This is always larger than one, contradicting the Poissonian hypothesis. Moreover, $R(T)$ is found to be an increasing function of $T$, so that it is no longer true that the fluctuations grow with time as $\sqrt{T}$.

Since our model takes into account only neutral and lethal mutations, without considering either advantageous mutations or slightly deleterious ones, we conclude that the violation of Poissonian statistics is not a decisive proof against the validity of the neutral hypothesis.

\section{PROPERTIES OF THE SEQUENCES}

We classify the nearly 12,000 sequences generated by our evolutionary algorithm in four classes, with the reminder that the identification of the ground state is only tentative for rejected sequences, as already discussed.

1. Selected sequences, belonging to the neutral network. Their number is a fraction $f=0.050$ of the total set.

2. Unstable sequences, $f=0.172$. Their lowest energy state coincides with $\mathbf{C}^{*}$, but the stability condition is not fulfilled. The rejection was made in most cases already after the first MC run, if the condition $\left\langle q\left(\mathbf{C}, \mathbf{C}^{*}\right)\right\rangle>0.75$ was not fulfilled, otherwise the sequence was studied in another MC run.

3. Slow folding sequences, $f=0.472$. For such sequences, no structure with energy lower than $E\left(\mathbf{C}^{*}, \mathbf{S}\right)$ was found, but the $\mathrm{MC}$ algorithm did not reach the target structure $\mathbf{C}^{*}$. In some cases $(f=0.061) \mathbf{C}^{*}$ was reached in the first MC run but not in the second one, while in most cases the rejection was made already after the first run.

4. Structurally mutated sequences, $f=0.306$. For such sequences the lowest energy structure $\mathbf{C}_{0}$, has lower energy than the target structure:

$$
E\left(\mathbf{C}_{0}, \mathbf{S}\right)<E\left(\mathbf{C}^{*}, \mathbf{S}\right)
$$


Before describing separately the properties of these classes of sequences, we show that the value of the $Z$ score is able to distinguish statistically the different classes. The $Z$ score [17 is used to evaluate the match between a sequence $\mathbf{S}$ and a structure $\mathbf{C}^{*}$ taken from a pool of alternative structures. It is defined as

$$
Z\left(\mathbf{C}^{*}, \mathbf{S}\right)=\frac{E\left(\mathbf{C}^{*}, \mathbf{S}\right)-\langle E(\mathbf{C}, \mathbf{S})\rangle}{\sqrt{\left\langle E^{2}(\mathbf{C}, \mathbf{S})\right\rangle-\langle E(\mathbf{C}, \mathbf{S})\rangle^{2}}},
$$

where the brackets denote average with respect to the ensemble of alternative structures at high temperature. The more negative $Z$ is, the better is the match between the sequence $\mathbf{S}$ and the structure $\mathbf{C}^{*}$ and the more stable is the structure $\mathbf{C}^{*}$, provided that it is really the lowest energy structure. This measure is often used in computer experiments of fold recognition [16,17.

Following Mirny and Shakhnovich 32], we use a simplified measure of the $Z$ score, considering as alternative structures only maximally compact structures and approximating their average energy and their variance with, respectively, the average energy and the variance of the set of all possible contacts (we take into account the fact that in the simple cubic lattice the only possible contacts are those between monomers of different parity). More precisely, our definition is

$$
Z^{\prime}\left(\mathbf{C}^{*}, \mathbf{S}\right)=\frac{E\left(\mathbf{C}^{*}, \mathbf{S}\right)-N c_{\max }\left[U\left(S_{i}, S_{j}\right)\right]}{N c_{\max } \sqrt{\left[U^{2}\left(S_{i}, S_{j}\right)\right]-\left[U\left(S_{i}, S_{j}\right)\right]^{2}}},
$$

where

$$
U\left(S_{i}, S_{j}\right)=\frac{\sum_{i j} P_{i j} U\left(S_{i}, S_{j}\right)}{\sum_{i j} P_{i j}}
$$

$P_{i j}$ is one if a contact between amino acids $i$ and $j$ is possible in some configurations, zero otherwise and $N c_{\max }$ is the number of contacts for maximally compact structures, $N c_{\max }=40$ for $N=36$ on the cubic lattice. $Z^{\prime}$ is a good approximation to the $Z$ score and it is very easy to compute numerically, without the need for a simulation at a high temperature.

We plot in figure 1 the distribution of the $Z$ score for the four classes of sequences. For the structurally mutated sequences we evaluated both the $Z$ score of the target structure $\mathbf{C}^{*}$ and the $Z$ score of the lowest energy structure found, $\mathbf{C}_{0}$. Note that the starting sequence $\mathbf{S}(0)$ has the lowest value of the $Z$ score, i.e. $Z=-1.22$.

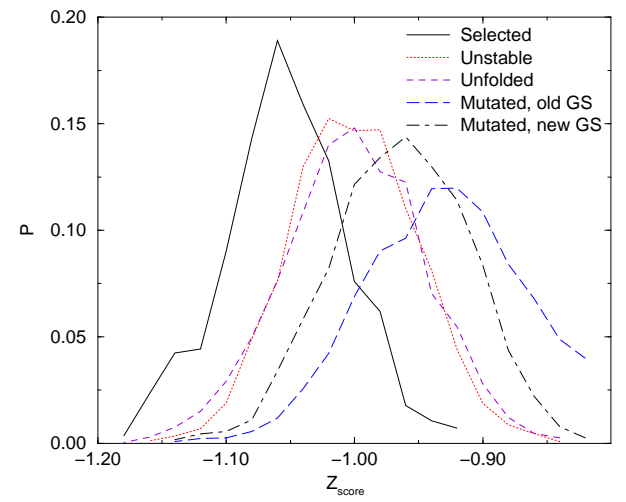

FIG. 4. Distribution of the $Z$ score for different classes of sequences.

The ranking of the $Z$ score for different classes is as expected on the basis of their stability. The most negative values of $Z$ are proper of selected sequences, which are most stable. Next come slow folding and unstable sequences. The $Z$ score of mutated ground states, $Z\left(\mathbf{C}_{0}, \mathbf{S}\right)$, are less negative. Last in this rank of stability comes the $Z\left(\mathbf{C}^{*}, \mathbf{S}\right)$ for the structurally mutated sequences. In this case, we are sure that $\mathbf{C}^{*}$ is not the ground state of the sequence.

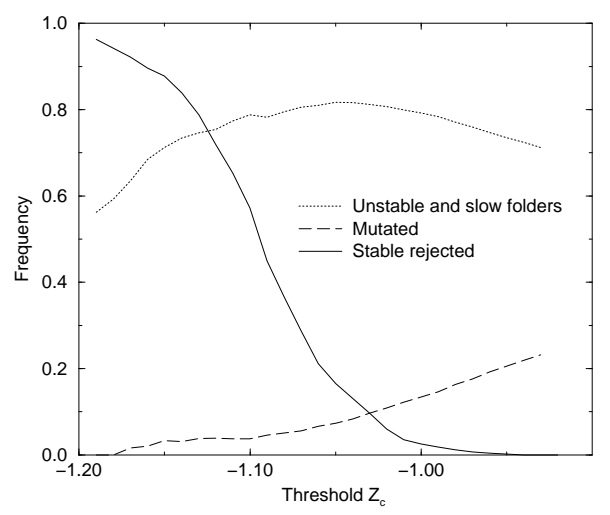

FIG. 5. Fraction of unstable, slow folding and mutated sequences that would be selected with a criterion based on a threshold value of the $Z$ score, $Z_{c}$. Solid line: fraction of sequences selected by our algorithm that would be rejected with the same criterion.

These results confirm that the evaluation of the $Z$ score is an efficient criterion to decide whether $\mathbf{C}^{*}$ is the ground state of a sequence $\mathbf{S}$. Nevertheless, the fact that the distributions of the $Z$ score relative to different classes have large overlaps should make one worry that the $Z$ score is not a precise criterion. In Fig.5 we report the results that we would get using a threshold value of $Z, Z_{c}$, as a criterion for fold recognition, instead of studying the sequences with Monte Carlo simulations. The dotted line represents the fraction of sequences that are unstable or 
slow folders according to our criterion and would be accepted with a criterion based on the $Z$ score. This goes from less than $60 \%$ for the most stringent threshold to a plateau value of about $80 \%$. We can say very little about this class of sequences. It includes sequences that are really of lower quality than selected sequences, sequences that are of the same quality but were not selected because of the uncertainties of the selection procedure and sequences which do not fold to the target state. The dashed line represents sequences whose ground state is surely different from the target one. Their fraction increases from zero to about $20 \%$ as the threshold becomes less stringent. Finally, the solid line represents sequences that would be selected with our criterion but not with the $Z$ score criterion, as a fraction of the total number of selected sequences. Our results indicate that a good choice for the threshold could be $Z_{c} \approx-1.07: 14 \%$ of the sequences selected with this criterion would also be selected with our criterion, $80.5 \%$ would be sequences that do not fulfill the stability or fast folding conditions and $5.5 \%$ would be sequences which have certainly a different ground state, but probably similar to the target one, since the $Z$ score of mutated sequences is correlated to the similarity between the new ground state and the target state (see below). About $29 \%$ of the sequences that our criterion selects would be discarded with the $Z$ score criterion. This number becomes much larger if the threshold is made more stringent. Thus, the criterion based on $Z$ accepts most sequences that we reject and rejects a large fraction of those that we select.

The distribution for the slow folding class is quite similar to that of the unstable class. This is not surprising, since it is well known that stability and fast folding are correlated in lattice heteropolymer models [33:34]. In particular, stability as we defined it requires a correlated energy landscape, which is considered a property of fast folding sequences. Thus these results encourage us in believing that the conditions we imposed and the algorithm to verify them were appropriate.

Interestingly, the distribution relative to $Z\left(\mathbf{C}_{0}, \mathbf{S}\right)$ lies to the right of the other ones, indicating that the stability of the structurally mutated ground states is rather low. This is not unexpected. In fact, structurally mutated sequences are only one point mutation apart from selected sequences, thus $\mathbf{C}^{*}$ should still have a low energy and should decreases the stability of $\mathbf{C}_{0}$. We shall comment further on this point in the conclusions.

The $Z$ score correlates well to the native overlap $\left\langle q\left(\mathbf{C}, \mathbf{C}^{*}\right)\right\rangle$ that we assumed as a measure of thermodynamic stability (Fig.6) for unstable sequences (correlation coefficient $r=-0.48$ ) and for mutated sequences (this is due to the fact that the overlap between the mutated ground state and the native state correlates with to the $Z$ score). No correlation is visible for selected sequences, which always have $\left\langle q\left(\mathbf{C}, \mathbf{C}^{*}\right)\right\rangle>0.9$. For unfolded sequences the measure of $\left\langle q\left(\mathbf{C}, \mathbf{C}^{*}\right)\right\rangle$ is not possible (see Fig.6).

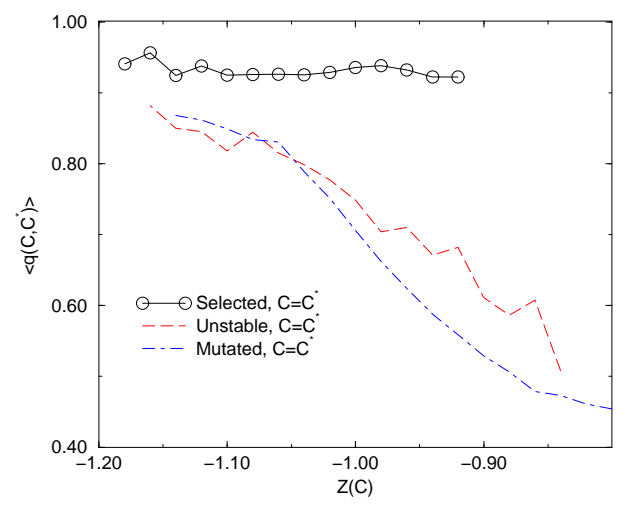

FIG. 6. Average value of the native overlap as a function of the $Z$ score $Z(C)$.

\section{A. Selected sequences}

With our criterion we accepted 566 sequences in six evolutive trajectories. Such sequences are both fast folding and thermodynamically stable. These properties are not typical of random sequences 23].

For selected sequences there is a significant correlation between the energy $E$ of a conformation and its overlap $q$ with the native state. In Fig. 7 we represent the 500 lowest energy configurations of three different sequences as points in the $(E, q)$ plane. For every sequence, all points fulfill the inequality

$$
1-E(\mathbf{C}, \mathbf{S}) / E\left(\mathbf{C}^{*}, \mathbf{S}\right) \geq \alpha(\mathbf{S})\left(1-q\left(\mathbf{C}, \mathbf{C}^{*}\right)\right) .
$$

The adimensional parameter $\alpha(\mathbf{S})$ is related to the energy gap of the ground state $\mathbf{C}^{*}$ of sequence $\mathbf{S}$, as defined by Shakhnovich and coworkers [34]. However, it characterizes more precisely the smoothness of the energy landscape. For the initial sequence we find $\alpha\left(\mathbf{S}_{0}\right)=0.23$. We did not measure $\alpha(\mathbf{S})$ for all selected sequences, but it appears from few examples that its value does not decrease during the evolution of the protein. It is not surprising that selected sequences exhibit a correlated energy landscape, since the condition of thermodynamic stability that we impose rules out sequences with misfolded structures of low energy. Moreover, our selected sequences are fast folders, and one should expect that such sequences have a correlated energy landscape, since the relation between thermodynamic stability, fast folding and smoothness of the energy landscape has long been discussed [24,23]. Furthermore, it has been found that models which cannot give rise to good folding sequences present weak correlations between $q$ and $E$ 35.28].

Consistently, it appears from our data that the folding time is correlated to the stability (it has a negative correlation with the $Z$ score and positive with the native overlap), but we are not able to quantify this effect, since our measure of the folding time, based only on two simulations, is too imprecise. 


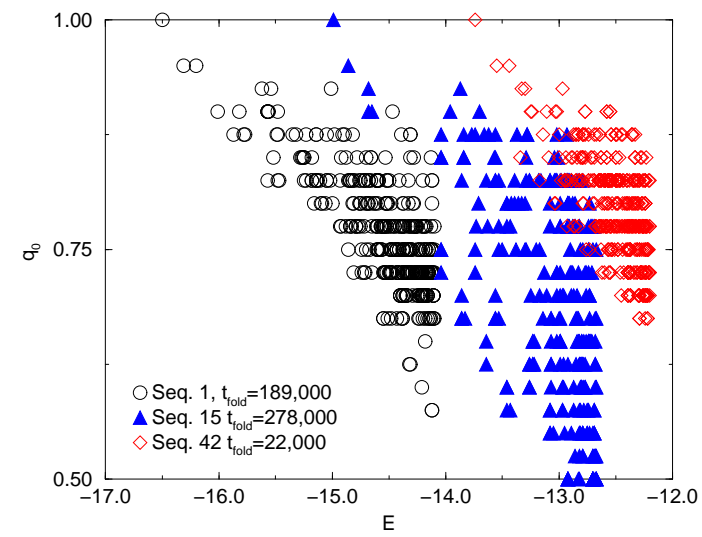

FIG. 7. Correlations between the energy and the similarity with the ground state for the 200 lowest energy structures of three selected sequences.

It is also interesting that for several sequences the en$\operatorname{ergy} E\left(\mathbf{C}^{*}, \mathbf{S}\right)$ is lower than for the starting sequence $\mathbf{S}^{*}$, although this has been obtained by minimizing the energy $E\left(\mathbf{C}^{*}, \mathbf{S}\right)$ in sequence space. The reason for this is that the minimization method requires that the composition of the sequence is kept fixed, while we do not impose this condition. However, the $Z$ score reaches its lowest value $Z=-1.22$ for the starting sequence $S^{*}$.

We observe that the $Z$ score of the target state, $Z\left(\mathbf{C}^{*}, \mathbf{S}\right)$, defines a complex landscape in sequence space, with valleys separated by barriers. This result is illustrated in Fig.8, where we show the $Z$ score of sequences in the neutral network as a function of the number of steps $l$ along the network, starting from $\mathbf{S}^{*}$.

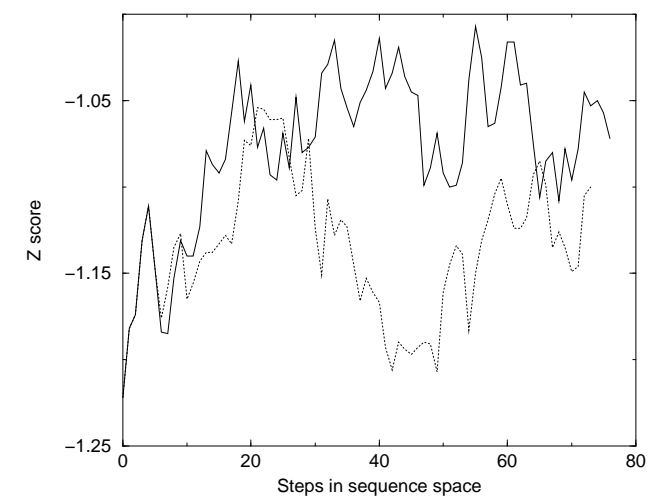

FIG. 8. $Z$ score of sequences in the neutral network as a function of the number of steps along the network, starting from $\mathbf{S}^{*}$, for two evolutionary trajectories.

The roughness of the energy landscape is consistent with the results reported in a recent preprint by Tiana et al. 20], who sampled the energy landscape in sequence space for a fixed structure using Monte Carlo simulations. The authors of [20] observed a hierarchy of clusters and superclusters of low energy sequences, with superclusters characterized by few fixed amino acids in key positions and not connected by neutral paths. This conclusion might at first seem at odd with the fact that we found connected neutral paths extended in sequence space, but there should be no contradiction between the present work and the results of ref. [20], since they deal with different questions. We studied a single neutral network asking whether it is possible to find in it pairs of sequences at any distance, while Tiana et al. 20] ask whether it is possible to find non homologous proteins which are in disconnected neutral networks and still fold to the same structure. It is possible that both answers are positive and that several extended but disconnected neutral networks exist for a given protein structure. Such a picture, if correct, implies that non homologous proteins sharing the same fold may have been originated either through convergent evolution, possibly on disconnected neutral networks, or through divergent evolution from a common anchestor on a single neutral network, but it is very difficult or even impossible to decide between these two possibilities on the basis of the sequence alone.

On the other hand, we should note that the definition of neutral path in our work is different than the one used in [20]. In fact, while in [20] is assumed that a path in sequence space is neutral if all sequences belonging to it have $Z$ score lower than a predetermined threshold, we accept only sequences for which we can show through Monte Carlo simulations that the target structure is the ground state, it is thermodynamically stable and easy to reach kinetically. The criterion adopted in 20] has the advantage of being computationally very efficient and it correlates well with our criterion. In several cases, however, the two criteria give different answers, as it is shown in Fig. 月, and it is possible that networks which are disconnected according to the $Z$ score criterion are found to be connected according to our criterion. Further study is necessary in order to assess the relevance of such possibility.

It is rather interesting that the complex energy landscape in sequence space offers an explanation for the large variations of the neutral mutation rate, $x(\mathbf{S})$. Since a more stable sequence can tolerate larger rearrangements without changing its ground state, one can expect that the fraction of neutral mutations from sequence $\mathbf{S}$ increases with the stability of the sequence. This expectation is in agreement with the results of ref. [20], where the stability is measured by the $Z$ score $Z\left(\mathbf{C}^{*}, \mathbf{S}\right)$ and neutrality of a mutation is recognized with a criterion based on the $Z$ score of the mutated sequence.

We study the correlation between the stability, measured either by the native overlap or by the $Z$ score, and the fraction of neutral neighbors $x(\mathbf{S})$. Since we did not measure $x(\mathbf{S})$, we have to rely on the trapping time $\tau_{t}$ spent by a trajectory on sequence $\mathbf{S}$. This variable is related to $x(\mathbf{S})$ through the geometric distribution $P_{x}(\tau)=$ $x(1-x)^{\tau-1}$ of average value $1 / x(\mathbf{S})$ (11). We thus estimate the correlation coefficient between the $Z$ score and $1 / x(\mathbf{S})$, using the relations $[Z(\mathbf{S}) / x(\mathbf{S})] \approx\left[Z(\mathbf{S}) \tau_{t}(\mathbf{S})\right]$, $[1 / x(\mathbf{S})] \approx\left[\tau_{t}(\mathbf{S})\right],\left[1 / x(\mathbf{S})^{2}\right] \approx 1 / 2\left[\tau_{t}^{2}(\mathbf{S})+\tau_{t}(\mathbf{S})\right]$, 
where the square brackets indicate average on the neutral network. This treatment neglects the fact that our criterion is subject to some arbitrariness, since we can not measure with high precision the native overlap $\langle q\rangle$ and the typical folding time upon which our criterion is founded. Thus, the correlation coefficient estimated in this way is underestimated. We find a correlation coefficient $r=0.20$ between the $Z$ score and $1 / x$ and $r=-0.21$ between the native overlap and $1 / x$. Although the estimate is not accurate, this study confirms the existence of correlations between the stability of the native state and the neutral mutation rate. We show the correlation between $Z$ and $\tau_{t}$ in Fig.9.
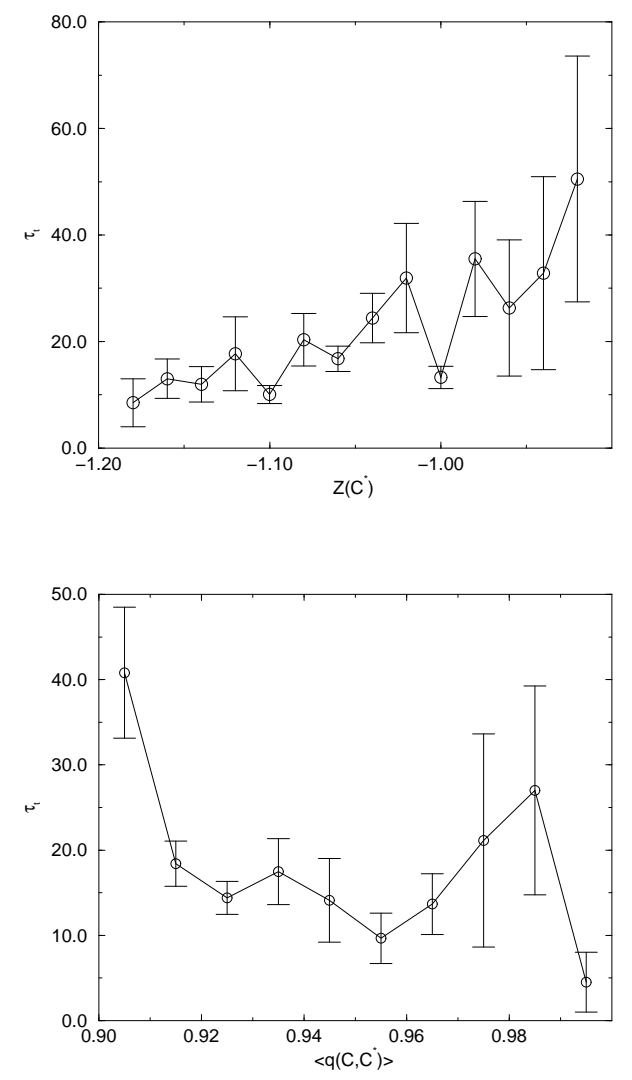

FIG. 9. Average value of $\tau_{t}$ for sequences of the neutral network as a function of the $Z$ score (upper panel) and of the average native overlap (lower panel).

The previous observation can be the basis for a quantitative explanation of the large fluctuations of the neutral mutation rate in this model and possibly in real proteins. It would be very interesting to investigate to which extent such fluctuations are responsible for the observed patterns of molecular evolution, which appear to be much more irregular than predicted on the basis of the simple "homogeneous" neutral theory.

\section{B. Unstable and slow folding sequences}

To these two classes belong all sequences that were rejected although no structure of energy lower than $\mathbf{C}^{*}$ was found. We do not know for which fraction of these sequences the ground state coincides with $\mathbf{C}^{*}$ and for which the ground state is changed. For unstable sequences $\mathbf{C}^{*}$ is the lowest energy structure found and it is reached in at least one of the two independent MC runs, but the stability condition is not fulfilled. The rejection was made already in the first MC run if we found $\langle q\rangle>0.75$. Thus we can not exclude that in the second run also the fast folding condition would fail. For slow folding sequences $\mathbf{C}^{*}$ was not reached either in the first or in the second $\mathrm{MC}$ run. The folding time for unstable sequences appears to be correlated to the native overlap $\langle q\rangle$, even if our data do not allow quantitative estimations.

\section{Structurally mutated sequences}

For sequences in this class we found putative ground states with energy lower than that of the target structure. A fraction $f=0.306$ of the examined sequences belongs to this class.

We first analyze the number $N_{c}^{0}$ of contacts in the mutated ground state. The distribution $P\left(N_{c}^{0}\right)$ is reminiscent of a bimodal distribution (see Fig. 10). As previously mentioned, the number of target contacts, $N_{c}^{*}=40$, is the largest possible for a sequence length of $N=36$ residues. The twin peaks at $N_{c}^{0}=37$ and at $N_{c}^{0}=40$ derive from target-like ground states, while the constraints of the lattice geometry are probably responsible for the depression of the values of $P(38)$ and $P(39)$. The broad peak at $N_{c}^{0}=34$ is close to the number of contacts expected for random sequences, although slightly higher. As mentioned above, in the case of a random contact potential with the same mean and variance as the one that we used and a Gaussian distribution, the number of contacts in the ground states ranges typically from 29 to 33 25].

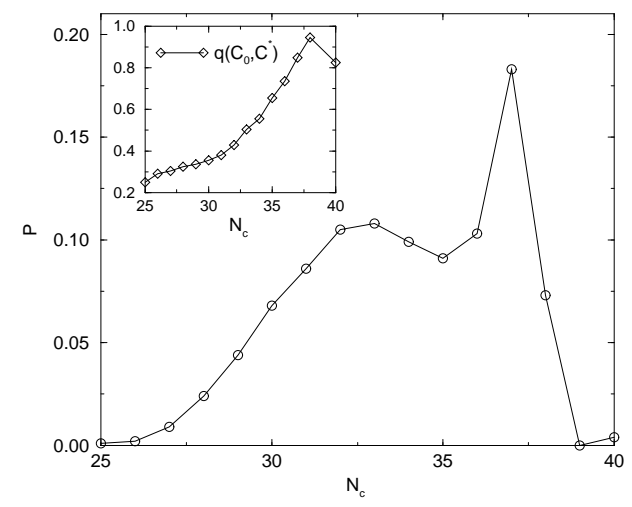


FIG. 10. Distribution of the number of contacts in the ground state, $N_{c}$, for all of the generated sequences. The peaks at high $N_{c}$ are related to target-like structures. Insert: correlation between the number of contacts and the overlap $q_{0}$ between target state and ground state.

The number of contacts is higher than for random sequences because the "native" contacts of $\mathbf{C}^{*}$ are advantageous even in the structurally mutated sequences. This is confirmed by the fact that there is a strong correlation correlation between the number of contacts $N_{c}^{0}$ and the overlap $q_{0}$ between the new ground state and the target state $\mathbf{C}^{*}$ The correlation coefficient is $r=0.80$. See insert in Fig.10. The two quantities are also correlated to the energy $E_{0}$ of the ground state: The more native-like the mutated ground state is, the more compact it is and the lower is its energy. The correlation coefficients are: $r=-0.31$ between $q_{0}$ and $E_{0}, r=-0.32\left(N_{c}^{0}\right.$ and $\left.E_{0}\right)$, $r=-0.54$ ( $q_{0}$ and $Z$ score $)$ and $r=-0.47\left(N_{c}^{0}\right.$ and $Z$ score). The strongest correlation observed is that between $q_{0}$ and the $Z$ score. The energy and the $Z$ score are weekly correlated $(r=0.36)$.

The distribution of $q_{0}$ is also bimodal (see Fig. 11). The peak at high $q$ is due to native-like structures and the broader peak at $q=0.3$ is close to (but still significantly higher than) the typical overlap between random structures, $q_{\text {ran }}=0.1$ [25] for chains of length $N=36$.

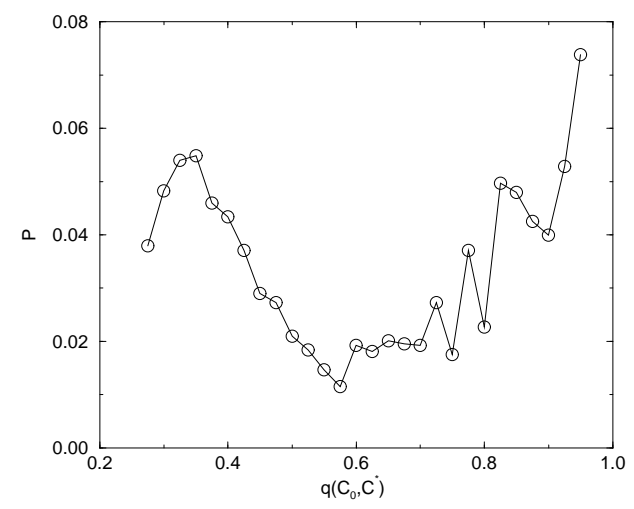

FIG. 11. Distribution of the overlap $q\left(\mathbf{C}^{*}, \mathbf{C}\right)$ between the target structure $\mathbf{C}^{*}$ and the ground-state of the sequences studied, C.

The overlap $q_{0}$ between the ground state and the target state is negatively correlated to the $Z$ score. This is shown in Fig.12, where all the structurally mutated sequences are represented in a scatter plot in the plane $\left(q_{0}, Z\right)$. Only structures which are very similar to the original native state have a $Z$ score in the range found for selected sequences. This result suggests that mutated ground states dissimilar from the original one are in most cases not stable enough to represent a new acceptable fold of the model protein. In other words, neutral networks of unrelated structures may lay far apart in sequence space. This feature of the model is consistent with the observa- tion that biological evolution conserves the native fold of proteins even when their function changes substantially [2, 3.

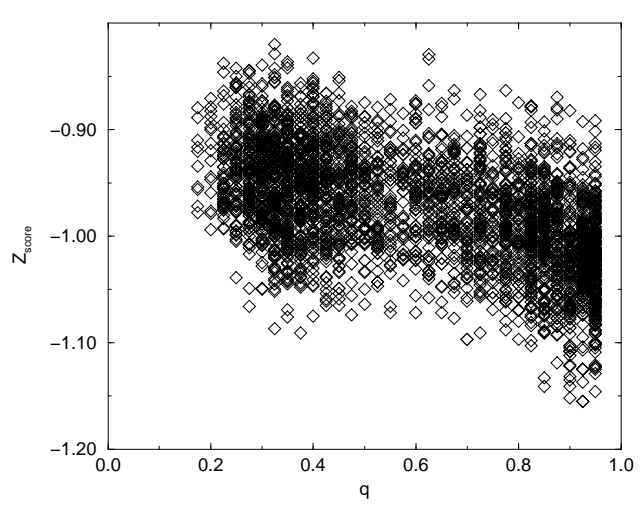

FIG. 12. Scattering plot showing the $Z$ score of the ground state as a function of its overlap with the target state.

\section{DISCUSSION}

We simulated evolution on neutral networks for a protein model with twenty amino acid types, contact energy functions and structures represented as self avoiding walks on the simple cubic lattice. Stability of the ground state is measured as the thermodynamic average of its overlap with alternative structures.

Our simulations show that neutral networks are extended in sequence space: pairs of sequences on the neutral network are almost as different as random sequences, even if they have exactly the same fold. This observation is consistent with what is known about protein evolution. In our 36 mer chains, residues in all positions could be substituted, even if the two positions in the core are much more difficult to change, consistently with the results of Ref. 30].

The neutral network that we studied turned out to be rather irregular: the fraction $x(\mathbf{S})$ of neutral mutations of a sequence $\mathbf{S}$ has large variations in sequence space. The value of $x(\mathbf{S})$ is positively correlated with the stability of the native state, evaluated either through the average native overlap $\left\langle q\left(\mathbf{C}, \mathbf{C}^{*}\right)\right\rangle$ or through the $Z$ score. This appears very reasonable: The more stable is a sequence to structure match, the less probable is that a mutation destabilizes it. This is also in agreement with the results of recent complementary studies 36,29. It thus seems that thermodynamic stability implies also stability with respect to mutations.

The fluctuations of $x(\mathbf{S})$ in sequence space are important for the evolutionary dynamics. If $x(\mathbf{S})$ is constant, Kimura's theory of neutral evolution predicts that the overlap in sequence space relaxes exponentially to the asymptotic value corresponding to random sequences, at a rate independent of the size of the biological population in which the evolution takes place. Moreover, the 
number of substitutions in a protein sequence during an evolutionary trajectory lasting $T$ generations is predicted to be a Poissonian variable of mean value $\mu x T$. In this case, the fluctuations of the value of this variable in different species evolving for a time $T$ should grow only as $\sqrt{T}$. This result would sustain the molecular clock hypothesis, according to which the substitution process can be used as a clock to date speciation events. However, the molecular clock hypothesis has been heatedly debated in the last decade, and it has been shown that the number of substitutions fluctuates much more than predicted in the "homogeneous" neutral theory [8]. This deviation from the prediction of Kimura's theory has been interpreted as an indication that in most cases protein evolution is not neutral but adaptive. We suggest that the irregularity of protein evolution could be an intrinsic property of the energy landscape of neutral networks of protein sequences. More stringent statistical tests should be designed to distinguish this situation from adaptive evolution, that undoubtedly occurs in many cases.

Selected sequences have a rather correlated energy landscape, which yields short folding times and high thermodynamic stability. The native overlap that we use as a measure of thermodynamic stability correlates well with the $Z$ score but it gives more information on the absence of states of low energy unrelated to the native state and favors a more correlated energy landscape.

Sequences whose ground state coincides with the native state may be discarded either due to the lack of thermodynamic stability or because they fold too slowly. Both classes of sequences have similar properties, since stability and folding time are related quantities.

For about $30 \%$ of the attempted mutations the resulting sequence has a ground state different than the original one. The overlap $q_{0}$ of this new ground state with the target state has a bimodal distribution, but only structures very similar to the target one appear to fulfill our criterion of thermodynamic stability. This is not surprising, since the target structure must conserve a low energy in the mutated sequence, so that it is able to destabilize the new ground state. Therefore, it seems that contacts between neutral networks of unrelated structures are very rare, if a stability condition is required. A similar conclusion has been suggested in a numerical study of the two dimensional HP model [14].

An implication of this result is that it is difficult to switch from a structure to a different one through point mutations corresponding to thermodynamically stable proteins. This could explain why evolution changes so rarely the fold of a protein, while it is possible to engineer protein sequences with as much as $60 \%$ similarity with a natural protein and completely different fold.

We studied a model which has the advantage of being reliably computable, but at the price of sacrificing possibly important ingredients. We discuss here the ones that we judge the most serious:

1. We use a simple lattice model. This choice was made due to the necessity of identifying the native state of each generated sequence, and this is feasible only for lattice models. Lattice models, although often criticized [37], have been recognized to capture some of the most relevant thermodynamic features of the folding process [38], such as the existence of a unique ground state and the cooperativity of the transition. However, they do not capture essential features of real proteins as for instance the existence of secondary structures.

2. We simulated the evolution of only one target structure. It would be interesting to see how our results change by changing the structure, and which properties of the structure (for instance compactness, locality of interactions and so on) are important to determine the neutral mutation rate. However, it was argued that the small number of folds occurring in natural proteins (at most some thousands) could be the ones corresponding to the largest number of sequences in sequence space [39], so that structures characterized by a large neutral set, even if they are not typical, could be the most interesting ones from the biological point of view.

3. The length of the sequences examined is short, so that there are only two core residues. Considering more core residues could impose more constraints on the evolution and reduce the rate of neutral evolution. It would thus be interesting to make the same study for longer sequences.

4. We did not represent biological activity in the present protein model. This might be obtained by imposing more constraints on the residues taking part to the active site.

5. In our model of evolution we assume that the environment remains fairly constant, so that the native structure favored by natural selection does not change throughout the evolution. This hypothesis is not unreasonable if the protein examined is an enzyme performing some chemical activity, since the cells possess a high homeostasis, i.e. they can maintain a stable chemical-physical internal environment despite large perturbations in the external environment. However, it is quite likely that some large ecological and climatic changes have been responsible for molecular substitutions for which the neutral theory, and our model in particular, do not apply \&].

6. We consider only point mutations, and not insertions and deletions, which also play an important role in evolution.

In our opinion, these limitations should not modify the qualitative picture. The existence of neutral networks, the variability of neutral mutation rates and the difficulty 
to reach through point mutations very different structures corresponding to stable proteins are features of our model that appear to be reflected also in the evolution of real proteins.

\section{ACKNOWLEDGMENTS}

We acknowledge interesting discussions with Peter Grassberger, Helge Frauenkron, Erwin Gerstner, Walter Nadler, Peter Schuster, Anna Tramontano, Tim Gibson, Erich Bornberg-Bauer, Guido Tiana, Ricardo Broglia and Eugene Shakhnovich. This work was conceived during the workshop on Protein Folding organized at the ISI Foundation, Torino, Italy, February 9-13 1998. Part of the work was made during the Euroconference on "Protein Folding and Structure Prediction" organized at the ISI Foundation, Torino, Italy, June 8-19, 1998. Computations were carried out at the HLRZ, Forschungszentrum Jülich.

[1] B. Rost, Prot. Eng. 12, 85-94 (1999).

[2] L. Holm and C. Sander, Science 273, 595-602 (1996).

[3] P. K. Qasba and S. Kumar, Crit. Rev. Biochem. Mol. Biol. 32, 255-306 (1997).

[4] B. Rost, Fol. Des. 2, S19-S24 (1997).

[5] The number of amino acid matches obtained by pairing two random sequences of the same length is given by the binomial distribution with $p=1 / 20$ if one assumes that the 20 amino acids have the same probability to occur. For sequences of length $N$ there will be on average $p N$ identical amino acids, with a variance $N p(1-p)$. For random sequences, $95 \%$ of pairwise comparisons yield a sequence identity between $1 \%$ and $9 \%$.

[6] M. Kimura, The neutral theory of molecular evolution (Cambridge University Press, 1983).

[7] J.L. King and T.H. Jukes, Science164, 788-800 (1969).

[8] J.H. Gillespie, The causes of molecular evolution (Oxford University Press, 1991).

[9] F.J. Ayala, Proc. Natl. Acad. Sci USA94, 7776-7783 (1997).

[10] This problem is much simpler than protein folding and can be studied through efficient and reliable algorithms. See, e.g., P. Schuster, W. Fontana, P.F. Stadler, and I.L. Hofacker, Proc. R. Soc. London B 255, 279-284 (1994); M.A. Huynen, P.F. Stadler and W. Fontana, Proc. Natl. Acad. Sci. USA 93, 397-401 (1996).

[11] E.I. Shakhnovich and A.M. Gutin, J. Theor. Biol. 149, 537-546 (1991).

[12] G. Tiana, R.A. Broglia, H.E. Roman, E. Vigezzi and E.I. Shakhnovich, J. Chem. Phys. 108, 757-761 (1998).

[13] V.I. Abkevich, A.M. Gutin and E.I. Shakhnovich, J. Chem. Phys. 101, 6052-6062 (1994).

[14] E. Bornberg-Bauer, Biophys. J. 73, 2393-2403 (1997).
[15] U. Bastolla, H.E. Roman and M. Vendruscolo, J. Theor. Biol. 200, 49-64 (1999).

[16] A. Babajide, I.L. Hofacker, M.J. Sippl and P.F. Stadler, Fol. Des.2, 5, 261 (1997).

[17] J.U. Bowie, R. Lüthy and D. Eisenberg, Science253, 164170 (1991).

[18] S. Govindarajan and R.A. Goldstein, Proteins 29, 461466 (1997).

[19] S. Govindarajan and R.A. Goldstein, Biopolymers 42, 427 (1997).

[20] G. Tiana, R.A. Broglia, and E.I. Shakhnovich, preprint cond-mat/9905309 (1999).

[21] S. Miyazawa and R.L. Jernigan, Macromolecules 18, 534552 (1985).

[22] D.M. Epstein, S.J. Benkovic, P.E. Wright, Biochemistry 34, 11037-11048 (1995).

[23] E.I. Shakhnovich and A.M. Gutin, Proc. Natl. Acad. Sci. USA 90, 7195-7199 (1993); E.I. Shakhnovich, Phys. Rev. Lett.72, 3907-3910 (1994).

[24] J.D. Bryngelson and P.G. Wolynes, Proc. Natl. Acad. Sci. USA 84, 7524-7528 (1987).

[25] U. Bastolla, unpublished result.

[26] A similar substitution scheme, but with different selection mechanism, was used in V.I. Abkevich, A.M. Gutin and E.I. Shakhnovich Proc. Natl. Acad. Sci. USA93, 839844 (1996).

[27] P. Grassberger, Phys. Rev.E 56, 3682 (1997).

[28] H. Frauenkron, U. Bastolla, E. Gerstner, P. Grassberger and W. Nadler, Phys. Rev. Lett. 80, 3149-3152 (1998); H. Frauenkron, U. Bastolla, E. Gerstner, P. Grassberger and W. Nadler, Proteins 32, 52-66 (1998).

[29] R.A. Broglia, G. Tiana, H.E. Roman, E. Vigezzi and E.I. Shakhnovich, Phys. Rev. Lett. 82, 4727-4730 (1999).

[30] L.A. Mirny and E.I. Shakhnovich, J. Mol. Biol. 291, 177196 (1999).

[31] E. Zuckerkandl and L. Pauling, in Horizons in Biochemistry, eds. M. Kasha and B. Pullman (Academic Press, New York, 1962).

[32] L. Mirny and E.I. Shakhnovich, J. Mol. Biol. 264, 11641179 (1996).

[33] D.K. Klimov and D. Thirumalai, Jour. Chem. Phys 109, 4119-4125 (1998).

[34] A. Sali, E.I. Shakhnovich and M. Karplus, Jour. Mol. Biol. 235, 1614-1636 (1994); A.R. Dinner, V. Abkevich, E.I. Shakhnovich and M. Karplus, Proteins 35, 34-40 (1999).

[35] M. Vendruscolo, R. Najmanovich and E. Domany, preprint (1999).

[36] M. Vendruscolo, A. Maritan and J. R. Banavar, Phys. Rev. Lett. 78, 3967 (1997).

[37] B. Honig, and F. Cohen, Fold. Des. 1, R17 (1996).

[38] E.I. Shakhnovich, Fold. Des. 1, R50 (1996).

[39] A.V. Finkelstein, A.M. Gutin and A.Y. Badretdinov, FEBS Letters 325, 23 (1993). 